\title{
Comparative study of SPWM and SVPWM modulation algorithm based on dual Fourier integral theory for high performance current source converter
}

\author{
Jing Bai ${ }^{1, a^{*}}$, Jian $\mathrm{Liu}^{1, \mathrm{~b}}$, Chaochao $\mathrm{Li}^{1, \mathrm{c}}$, Yang $\mathrm{Li}^{1, \mathrm{~d}}$ and Huade $\mathrm{Li}^{2, \mathrm{e}}$ \\ ${ }^{1}$ College of Electrical Information Engineering, Beihua University, China \\ ${ }^{2}$ College of Mechanical Engineering, University of Science \& Technology Beijing, China \\ ajlbyj@163.com, bbeihualiujian@163.com, ${ }^{\mathrm{C}}$ licoco2040@163.com \\ d1361390822@qq.com, ${ }^{e} 1730137422 @ q q . c o m$
}

Keywords: Double Fourier integral theory; Current type inverter; SPWM; SVPWM Spectrum;THD ;WTHD.

Abstract. SPWM and SVPWM modulation methods are established based on the dual Fourier integral theory and the SPWM and THD modulation algorithms are compared. The results show that the SVPWM modulation algorithm can make the current frequency converter have more excellent output characteristics, which provides a theoretical basis for the practical application of the current mode converter.

\section{Topology and Modulation Technology of Current Transducer}

Topology of Current Transducer. The current type frequency converter is the most notable feature of the topology structure, which is used as the energy storage element in the DC port; To suppress the harmonic disturbance of the grid side, we use LC filter in the input port; The output port uses C filter to filter out the harmonic component caused by the modulation. Its topological structure is shown in Fig. 1.

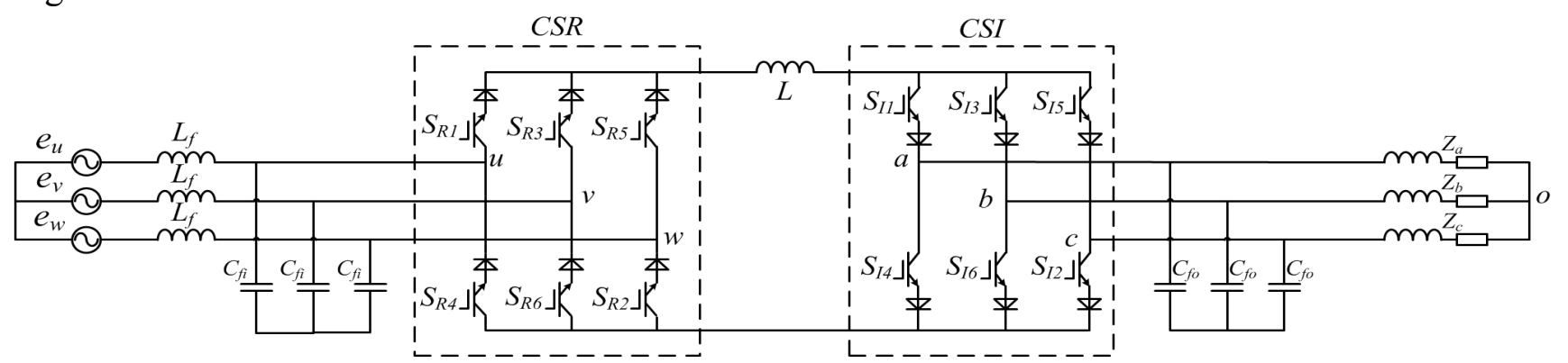

Fig. 1. Topological structure of three-phase current transducer

Technology of Current mode converter modulation technology. Many experts and scholars pay attention to the most important research areas of frequency converter and modulation technology. After decades of continuous development and improvement many outstanding and novel modulation technology are constantly put forward and applied. At present, there are four main types of modulation methods: specific harmonic elimination (SHE), trapezoidal pulse width modulation (TPWM), sinusoidal pulse width modulation (SPWM) and space vector modulation (SVPWM).In the above modulation algorithms, SPWM and SVPWM have been widely used in the best modulation performance. In this paper, we focus on the research of these two kinds of modulation algorithms, and show the theoretical foundation for the practical application of the current transducer.

\section{Double Fourier integral theory and performance index}

Double Fourier integral theory. We can use Double Fourier integral theory to analyze the harmonic of the inverter, and for a two variable function, the harmonic components and the form of the harmonic components can be written using the Fourier integral theory.. Double Fourier integral formula is as follows 


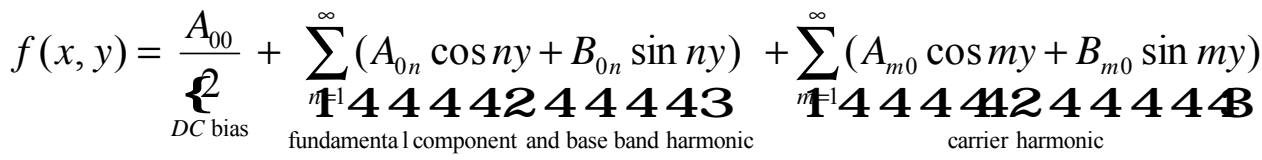

$$
\begin{aligned}
& +\sum_{m=1}^{\infty} \sum_{n=-\infty}^{\infty}\left[A_{m n} \cos (m x+n y)+B_{m n} \sin (m x+n y)\right] \\
& 14=444444244444444 B \\
& \text { side band harmonics }
\end{aligned}
$$

Where

$$
\begin{aligned}
& A_{m n}=\frac{1}{2 \pi^{2}} \int_{-\pi}^{\pi} \int_{-\pi}^{\pi} f(x, y) \cos (m x+n y) d x d y \\
& B_{m n}=\frac{1}{2 \pi^{2}} \int_{-\pi}^{\pi} \int_{-\pi}^{\pi} f(x, y) \sin (m x+n y) d x d y
\end{aligned}
$$

Or expressed in the plural form as follows

$$
C_{m n}=A_{m n}+j B_{m n}=\frac{1}{2 \pi^{2}} \int_{-\pi}^{\pi} \int_{-\pi}^{\pi} f(x, y) e^{j(m x+n y)} d x d y
$$

Here $x(t)=\omega_{c t}+\theta_{c}, y(t)=\omega_{0 t}+\theta_{0}, \omega_{c}$ is carrier frequency, $\theta_{c}$ is carrier phase offset angle. $\omega_{0}$ is fundamental angular frequency, $\theta_{c}$ is fundamental phase offset.

We can see any one of the pulse width modulation waveforms can be composed of five parts: DC bias, the fundamental component, the base band, the carrier wave and the side band by the double Fourier integral Eq. 1. Therefore, the double Fourier integral equation can be clearly analyzed in the different modulation algorithm under the output current waveform of various harmonic content and characteristics.

Performance index. Due to the inherent characteristics of the power electronic devices, the input and output waveforms are always accompanied by the inevitable harmonics. We define a variety of performance indicators to measure the severity of these harmonics to quantify the harmonic distortion. We can assume a periodic function of the current mode inverter output current is $i(t)$ and Cycle is $T$. Current mode converter can control the output of the current amount, so it can be expressed by Fourier series

$$
i(t)=I_{0}+I_{1} \cos \omega_{1} t+I_{2} \cos 2 \omega_{1} t+I_{3} \cos 2 \omega_{1} t+\cdots+I_{n} \cos n \omega_{1} t
$$

Here, the fundamental frequency is $\omega_{1}$.

THD is defined current total harmonic distortion

$$
T H D=\sqrt{\left(\frac{2 I_{0}}{I_{1}}\right)^{2}+\sum_{n=2}^{\infty}\left(\frac{I_{n}}{I_{1}}\right)^{2}}
$$

WTHD is defined weighted current total harmonic distortion

$$
W T H D=\frac{\sqrt{\sum_{n=2}^{\infty}\left(\frac{I_{n}}{n}\right)^{2}}}{I_{1}}
$$

Here, $I_{0}$ represents a straight flow, $I_{n}\left(\mathrm{n}=1,2, \ldots\left(I_{1}\right.\right.$ Fundamental $)$ representing the amplitude of the $\mathrm{n}$ wave. 


\section{A Comparative Study of SPWM and SVPWM Modulation Algorithms}

Triangular Carrier Symmetry Rule Sampling SPWM. The triangular carrier symmetry rule sampling SPWM is sampled at the positive or negative peak time of the carrier and the sampling value is kept constant in the carrier period. The asymmetric regular sampling SPWM is sampled at a positive or negative peak time in each half of the carrier cycle. From Fig. 2, we can show two sampling methods.

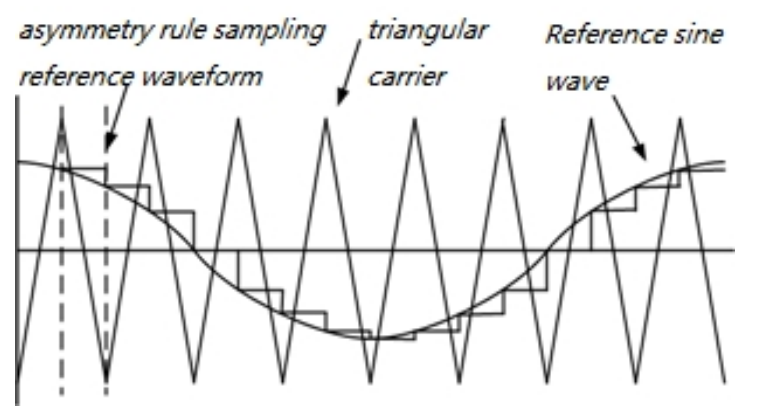

(a) Triangular carrier symmetry rule sampling SPWM

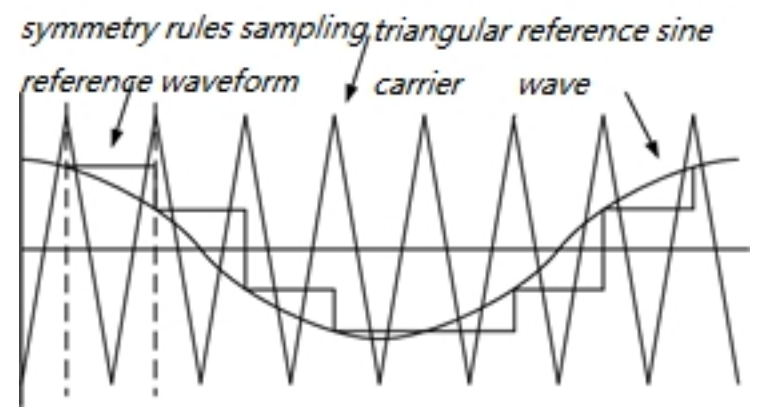

(b) Asymmetric sampling SPWM

Fig. 2. Triangular carrier sampling SPWM

Using double Fourier integral theory to analyze triangular carrier symmetry rule of the sampling SPWM.

$$
\begin{aligned}
A_{m n} & \left.+j B_{m n}=\frac{1}{2 \pi^{2}} \int_{-\pi}^{\pi} \int_{-\pi}^{-\frac{\pi}{2}(1+M c a s y)}-I_{d c} \cdot e^{j\left[\left(m+n \frac{\omega_{0}}{\omega_{c}}\right) x+n y\right.}\right] d x d y+\frac{1}{2 \pi^{2}} \int_{-\pi}^{\pi} \int_{-\frac{\pi}{2}(1+M c a s y)}^{\frac{\pi}{2}(1+M c a s y)} I_{d c} \cdot e^{j\left[\left(m+n \frac{\omega_{0}}{\omega_{c}}\right) x+n y\right.} d x d y \\
+ & \frac{1}{2 \pi^{2}} \int_{-\pi}^{\pi} \int_{\frac{\pi}{2}(1+M c a s y)}^{\pi}-I_{d c} \cdot e^{j\left[\left(m+n \frac{\omega_{0}}{\omega_{c}}\right) x+n y\right]} d x d y
\end{aligned}
$$

We can obtain single-phase half bridge phase current harmonics solutions by through the calculation of Eq. (8).

$$
\begin{aligned}
I(t) & =\frac{4 I_{d c}}{\pi} \sum_{n=1}^{\infty} \frac{1}{n \frac{\omega_{0}}{\omega_{c}}} J_{n}\left(n \frac{\omega_{0}}{\omega_{c}} \frac{\pi}{2} M\right) \sin \left[\left(n \frac{\omega_{0}}{\omega_{c}}+n\right) \frac{\pi}{2}\right] \cos \left[n\left(\omega_{0} t+\theta_{0}\right)\right] \\
& +\frac{4 I_{d c}}{\pi} \sum_{m=1}^{\infty} \frac{1}{m} J_{0}\left(m \frac{\pi}{2} M\right) \sin \left[\frac{\pi}{2} m\right] \cos \left[m\left(\omega_{0} t+\theta_{0}\right)\right] \\
& +\frac{4 I_{d c}}{\pi} \sum_{m=1}^{\infty} \sum_{\substack{n=-\infty \\
n \neq 0}}^{\infty} \frac{1}{m+n \frac{\omega_{0}}{\omega_{c}}} J_{n}\left[\left(m+n \frac{\omega_{0}}{\omega_{c}}\right) \frac{\pi}{2} M\right] \sin \left[\left(m+n \frac{\omega_{0}}{\omega_{c}}+n\right) \frac{\pi}{2}\right] \cos \left[m\left(\omega_{c} t+\theta_{c}\right)+n\left(\omega_{0} t+\theta_{0}\right)\right]
\end{aligned}
$$

The harmonic content in three-phase full bridge circuit

$$
\begin{aligned}
Y_{a}(t) & =\frac{1}{2}\left[X_{1}(t)-X_{2}(t)\right]=\frac{4 I_{d c}}{\pi} \sum_{n=1}^{\infty} \frac{1}{n \frac{\omega_{0}}{\omega_{c}}} \cdot J_{n}\left(n \frac{\omega_{0}}{\omega_{c}} \frac{\pi}{2} M\right) \sin \left[\left(n \frac{\omega_{0}}{\omega_{c}}+n\right) \frac{\pi}{2}\right] \sin \left(n \frac{\pi}{3}\right) \cos \left[n \omega_{0} t-n \frac{\pi}{3}+\frac{\pi}{2}\right] \\
& +\frac{4 I_{d c}}{\pi} \sum_{m=1}^{\infty} \sum_{n=-\infty}^{\infty} \frac{1}{n \neq 0} m+n \frac{\omega_{0}}{\omega_{c}} J_{n}\left[\left(m+n \frac{\omega_{0}}{\omega_{c}}\right) \frac{\pi}{2} M\right] \sin \left[\left(m+n \frac{\omega_{0}}{\omega_{c}}+n\right) \frac{\pi}{2}\right] \\
& \times \sin \left(n \frac{\pi}{3}\right) \cos \left[m \omega_{c} t+n\left(\omega_{0} t-\frac{\pi}{3}\right)+\frac{\pi}{2}\right]
\end{aligned}
$$




$$
\begin{aligned}
Y_{b}(t) & =\frac{1}{2}\left[X_{2}(t)-X_{3}(t)\right]=\frac{4 I_{d c}}{\pi} \sum_{n=1}^{\infty} \frac{1}{n \frac{\omega_{0}}{\omega_{c}}} \cdot J_{n}\left(n \frac{\omega_{0}}{\omega_{c}} \frac{\pi}{2} M\right) \sin \left[\left(n \frac{\omega_{0}}{\omega_{c}}+n\right) \frac{\pi}{2}\right] \sin \left(n \frac{\pi}{3}\right) \cos \left[n \omega_{0} t-n \frac{\pi}{3}+\frac{\pi}{2}\right] \\
& +\frac{4 I_{d c}}{\pi} \sum_{m=1}^{\infty} \sum_{n=-\infty}^{\infty} \frac{1}{m+n \frac{\omega_{0}}{\omega_{c}}} J_{n}\left[\left(m+n \frac{\omega_{0}}{\omega_{c}}\right) \frac{\pi}{2} M\right] \sin \left[\left(m+n \frac{\omega_{0}}{\omega_{c}}+n\right) \frac{\pi}{2}\right] \\
& \times \sin \left(n \frac{\pi}{3}\right) \cos \left[m \omega_{c} t+n\left(\omega_{0} t-\frac{\pi}{3}\right)+\frac{\pi}{2}\right] \\
Y_{c}(t) & =\frac{1}{2}\left[X_{3}(t)-X_{1}(t)\right]=\frac{4 I_{d c}}{\pi} \sum_{n=1}^{\infty} \frac{1}{n \frac{\omega_{0}}{\omega_{c}}} \cdot J_{n}\left(n \frac{\omega_{0}}{\omega_{c}} \frac{\pi}{2} M\right) \sin \left[\left(n \frac{\omega_{0}}{\omega_{c}}+n\right) \frac{\pi}{2}\right] \sin \left(n \frac{\pi}{3}\right) \cos \left[n \omega_{0} t-n \frac{\pi}{3}+\frac{\pi}{2}\right] \\
& +\frac{4 I_{d c}}{\pi} \sum_{m=1}^{\infty} \sum_{n=-\infty}^{\infty} \frac{1}{n \neq 0} m+n \frac{\omega_{0}}{\omega_{c}} J_{n}\left[\left(m+n \frac{\omega_{0}}{\omega_{c}}\right) \frac{\pi}{2} M\right] \sin \left[\left(m+n \frac{\omega_{0}}{\omega_{c}}+n\right) \frac{\pi}{2}\right] \\
& \times \sin \left(n \frac{\pi}{3}\right) \cos \left[m \omega_{c} t+n\left(\omega_{0} t-\frac{\pi}{3}\right)+\frac{\pi}{2}\right]
\end{aligned}
$$

The complete harmonic solution of the phase current is obtained by using the symmetry rule sampling SPWM method.

$$
\begin{aligned}
I_{a}(t) & =Y_{a}(t)-Y_{b}(t)-Y_{c}(t)=\frac{4 I_{d c}}{\pi} \sum_{n=1}^{\infty} \frac{1}{n \frac{\omega_{0}}{\omega_{c}}} \cdot J_{n}\left(n \frac{\omega_{0}}{\omega_{c}} \frac{\pi}{2} M\right) \sin \left[\left(n \frac{\omega_{0}}{\omega_{c}}+n\right) \frac{\pi}{2}\right] \sin \left(n \frac{\pi}{3}\right) \\
& \times\left\{2 \sin \left[n \omega_{0} t+\frac{\pi}{2}\right] \sin \left(n \frac{\pi}{3}\right)+\cos \left(n \omega_{0} t+\frac{\pi}{2}\right)\right\}+\frac{4 I_{d c}}{\pi} \sum_{\substack{m=1 \\
n}}^{\infty} \sum_{\substack{n \neq 0 \\
n \neq 0}}^{\infty} \frac{1}{m+n \frac{\omega_{0}}{\omega_{c}}} J_{n}\left[\left(m+n \frac{\omega_{0}}{\omega_{c}}\right) \frac{\pi}{2} M\right] \\
& \times \sin \left[\left(m+n \frac{\omega_{0}}{\omega_{c}}+n\right) \frac{\pi}{2}\right] \sin \left(n \frac{\pi}{3}\right)\left[2 \sin \left(m \omega_{c} t+n \omega_{0} t+\frac{\pi}{2}\right) \sin \left(n \frac{\pi}{3}\right)+\cos \left(m \omega_{c} t+n \omega_{0} t+\frac{\pi}{2}\right)\right]
\end{aligned}
$$

From Fig. 3 we can obtain the phase current spectrum of the SPWM under the triangular carrier symmetry rule when Modulation ratio $\mathrm{M}=0.9$, Bobbi, $\mathrm{THD}=60.75 \%$, $\mathrm{WTHD}=2.05 \%$.

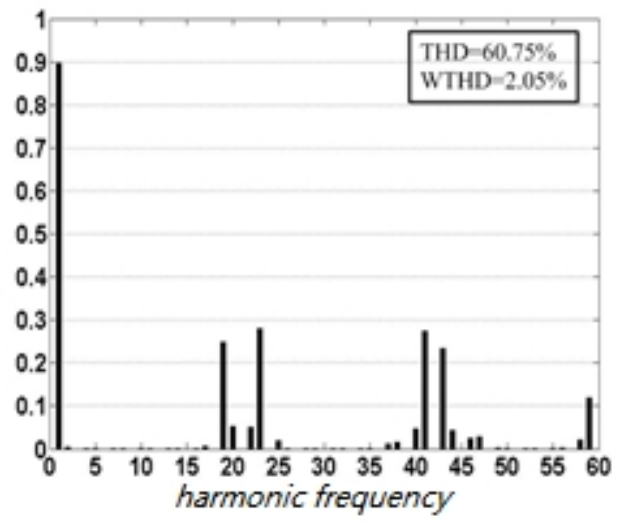

Fig. 3. The SPWM phase current spectrum of the three phase full bridge symmetrical regular sampling

Asymmetric sampling SPWM. Using the double Fourier integral theory to solve the harmonic component expression. 


$$
\begin{aligned}
A_{m n}+j B_{m n} & =\frac{1}{2 \pi^{2}} \int_{-\pi}^{\pi} \int_{-\pi}^{-\frac{\pi}{2}(1+M c a s y)}-I_{d c} \cdot e^{j\left\{m x+n\left[y+\frac{\omega_{0}}{\omega_{c}}\left(x+\frac{\pi}{2}\right)\right]\right\}} d x d y+\frac{1}{2 \pi^{2}} \int_{-\pi}^{\pi} \int_{-\frac{\pi}{2}(1+M c a s y)}^{0} I_{d c} \cdot e^{j\left\{m x+n\left[y+\frac{\omega_{0}}{\omega_{c}}\left(x+\frac{\pi}{2}\right)\right]\right\}} d x d y \\
& +\frac{1}{2 \pi^{2}} \int_{-\pi}^{\pi} \int_{0}^{\frac{\pi}{2}(1+M c a s y)}-I_{d c} \cdot e^{j\left\{m x+n\left[y+\frac{\omega_{0}}{\omega_{c}}\left(x+\frac{\pi}{2}\right)\right]\right\}} d x d y \\
& +\frac{1}{2 \pi^{2}} \int_{-\pi}^{\pi} \int_{\frac{\pi}{2}(1+M c a s y)}^{\pi}-I_{d c} \cdot e^{j\left\{m x+n\left[y+\frac{\omega_{0}}{\omega_{c}}\left(x+\frac{\pi}{2}\right)\right]\right\}} d x d y
\end{aligned}
$$

The calculation results show the single-phase half bridge phase current harmonic solution .Such as

$$
\begin{aligned}
I(t) & =\frac{4 I_{d c}}{\pi} \sum_{n=1}^{\infty} \frac{1}{n \frac{\omega_{0}}{\omega_{c}}} J_{n}\left(n \frac{\omega_{0}}{\omega_{c}} \frac{\pi}{2} M\right) \sin \left(n \frac{\pi}{2}\right) \cos \left[n\left(\omega_{0} t+\theta_{0}\right)\right] \\
& +\frac{4 I_{d c}}{\pi} \sum_{m=1}^{\infty} \frac{1}{m} J_{0}\left(m \frac{\pi}{2} M\right) \sin \left[\frac{\pi}{2} m\right] \cos \left[m\left(\omega_{0} t+\theta_{0}\right)\right] \\
& +\frac{4 I_{d c}}{\pi} \sum_{m=1}^{\infty} \sum_{\substack{n=-\infty \\
n \neq 0}}^{\infty} \frac{1}{m+n \frac{\omega_{0}}{\omega_{c}}} J_{n}\left[\left(m+n \frac{\omega_{0}}{\omega_{c}}\right) \frac{\pi}{2} M\right] \sin \left[(m+n) \frac{\pi}{2}\right] \cos \left[m\left(\omega_{c} t+\theta_{c}\right)+n\left(\omega_{0} t+\theta_{0}\right)\right]
\end{aligned}
$$

The following of the output current of three-phase full bridge harmonic solution for

$$
\begin{aligned}
Y_{a}(t) & =\frac{1}{2}\left[X_{1}(t)-X_{2}(t)\right]=\frac{4 I_{d c}}{\pi} \sum_{n=1}^{\infty} \frac{1}{n \frac{\omega_{0}}{\omega_{c}}} \cdot J_{n}\left(n \frac{\omega_{0}}{\omega_{c}} \frac{\pi}{2} M\right) \sin \left(n \frac{\pi}{2}\right) \sin \left(n \frac{\pi}{3}\right) \cos \left[n \omega_{0} t-n \frac{\pi}{3}+\frac{\pi}{2}\right] \\
& +\frac{4 I_{d c}}{\pi} \sum_{m=1}^{\infty} \sum_{n=-\infty}^{\infty} \frac{1}{n+n} J_{n}\left[\left(m+n \frac{\omega_{0}}{\omega_{c}}\right) \frac{\pi}{2} M\right] \sin \left[(m+n) \frac{\pi}{2}\right] \\
& \times \sin \left(n \frac{\pi}{3}\right) \cos \left[m \omega_{c} t+n\left(\omega_{0} t-\frac{\pi}{3}\right)+\frac{\pi}{2}\right] \\
Y_{b}(t) & =\frac{1}{2}\left[X_{2}(t)-X_{3}(t)\right]=\frac{4 I_{d c}}{\pi} \sum_{n=1}^{\infty} \frac{1}{n \frac{\omega_{0}}{\omega_{c}}} \cdot J_{n}\left(n \frac{\omega_{0}}{\omega_{c}} \frac{\pi}{2} M\right) \sin \left(n \frac{\pi}{2}\right) \sin \left(n \frac{\pi}{3}\right) \cos \left[n \omega_{0} t-n \pi+\frac{\pi}{2}\right] \\
& +\frac{4 I_{d c}}{\pi} \sum_{m=1}^{\infty} \sum_{n=-\infty}^{\infty} \frac{1}{m+n} J_{n}\left[\left(m+n \frac{\omega_{0}}{\omega_{c}} J_{c}\left[\frac{\pi}{2} M\right] \sin \left[(m+n) \frac{\pi}{2}\right]\right.\right. \\
& \times \sin \left(n \frac{\pi}{3}\right) \cos \left[m \omega_{c} t+n\left(\omega_{0} t-\pi\right)+\frac{\pi}{2}\right] \\
& +\frac{4 I_{d c}}{\pi} \sum_{m=1}^{\infty} \sum_{n=-\infty}^{\infty} \frac{1}{n \neq 0} m+n \frac{\omega_{0}}{\omega_{c}} J_{n}\left[\left(m+n \frac{\omega_{0}}{\omega_{c}}\right) \frac{\pi}{2} M\right] \sin \left[(m+n) \frac{\pi}{2}\right] \\
& \times \sin \left(n \frac{\pi}{3}\right) \cos \left[m \omega_{c} t+n\left(\omega_{0} t+\frac{\pi}{3}\right)+\frac{\pi}{2}\right] \\
Y_{c}(t) & =\frac{1}{2}\left[X_{3}(t)-X_{1}(t)\right]=\frac{4 I_{d c}}{\pi} \sum_{n=1}^{\infty} \frac{1}{n \frac{\omega_{0}}{\omega_{c}}} \cdot J_{n}\left(n \frac{\omega_{0}}{\omega_{c}} \frac{\pi}{2} M\right) \sin \left(n \frac{\pi}{2}\right) \sin \left(n \frac{\pi}{3}\right) \cos \left[n \omega_{0} t+n \frac{\pi}{3}+\frac{\pi}{2}\right] \\
&
\end{aligned}
$$


The complete harmonic solution of the phase current is obtained by using the asymmetric rule sampling SPWM method

$$
\begin{aligned}
I_{a}(t) & =Y_{a}(t)-Y_{b}(t)-Y_{c}(t)=\frac{4 I_{d c}}{\pi} \sum_{n=1}^{\infty} \frac{1}{n \frac{\omega_{0}}{\omega_{c}}} \cdot J_{n}\left(n \frac{\omega_{0}}{\omega_{c}} \frac{\pi}{2} M\right) \sin \left(n \frac{\pi}{2}\right) \sin \left(n \frac{\pi}{3}\right) \\
& \times\left\{2 \sin \left[n \omega_{0} t+\frac{\pi}{2}\right] \sin \left(n \frac{\pi}{3}\right)+\cos \left(n \omega_{0} t+\frac{\pi}{2}\right)\right\}+\frac{4 I_{d c}}{\pi} \sum_{\substack { m=1 \\
\begin{subarray}{c}{n=-\infty \\
n \neq 0{ m = 1 \\
\begin{subarray} { c } { n = - \infty \\
n \neq 0 } }\end{subarray}}^{\infty} \frac{1}{m+n \frac{\omega_{0}}{\omega_{c}}} J_{n}\left[\left(m+n \frac{\omega_{0}}{\omega_{c}}\right) \frac{\pi}{2} M\right] \\
& \times \sin \left[(m+n) \frac{\pi}{2}\right] \sin \left(n \frac{\pi}{3}\right)\left[2 \sin \left(m \omega_{c} t+n \omega_{0} t+\frac{\pi}{2}\right) \sin \left(n \frac{\pi}{3}\right)+\cos \left(m \omega_{c} t+n \omega_{0} t+\frac{\pi}{2}\right)\right]
\end{aligned}
$$

From Fig. 4 we can obtain the phase current spectrum of the SPWM under the asymmetric rule of the triangular carrier when Modulation ratio $\mathrm{M}=0.9$, Bobbi, $\mathrm{THD}=60.04 \%$, $\mathrm{WTHD}=2.02 \%$.

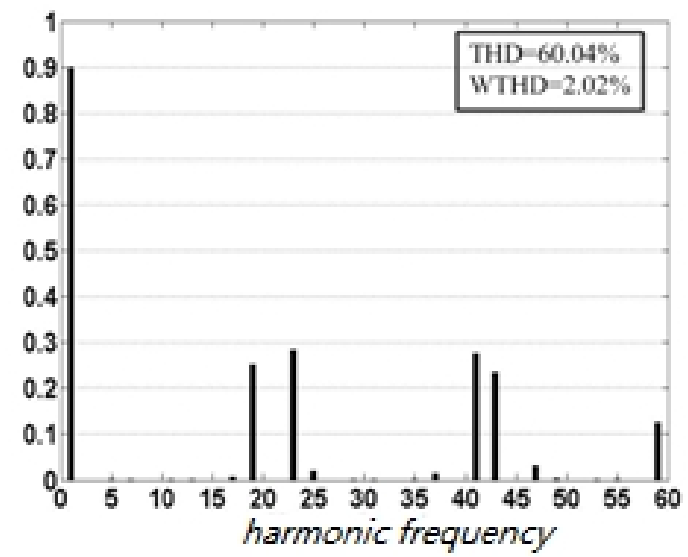

Fig. 4. The SPWM phase current spectrum of the three phase full bridge asymmetric regular sampling

Space vector modulation SVPWM. Current mode inverter SVPWM modulation technology has 9 kinds of switches, in which the SC7, SC8 and SC9 output terminal are defined as zero vector. The other 6 working states are called active vectors, where each valid vector interval is 60 .Figure 9 shows the current space vector map of the 5 vector in the space plane.

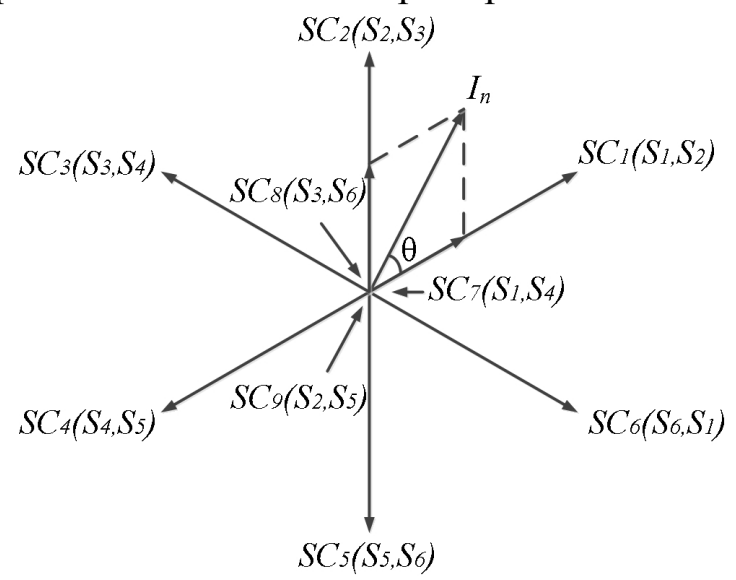

Fig. 5. Current space vector diagram

According to the sequence of SVPWM method, the mathematical expressions of the output current can be obtained by the geometric sum as follows

$$
\dot{I}_{n}=I_{n} \angle \theta_{0}=\frac{T_{S C_{1}}}{T / 2} S C_{1}+\frac{T_{S C_{2}}}{T / 2} S C_{2}
$$


On the equation corresponding to each of the switching intervals, which the action time is the time, for the time of action. The effective vector of target vector angle and the output values are available through two adjacent with a zero vector synthesis. Zero vector can be satisfied by the existence of zero vector, although the target output no value.

In conclusion, the following of the SVPWM harmonic component representation for

$$
A_{m n}+j B_{m n}=\frac{1}{2 \pi^{2}} \sum_{n=1}^{6} \int_{y_{x}(n)}^{y_{s}(n)} \int_{x_{x}(n)}^{x_{s}(n)} 2 I_{d c} \cdot e^{j(m x+n y)} d x d y
$$

Finally from Fig. 6 we can obtain the SVPWM phase current spectrum when THD $=52.28 \%$, WTHD $=1.75 \%$ under the three-phase full bridge circuit by a lot of calculation and simplification.

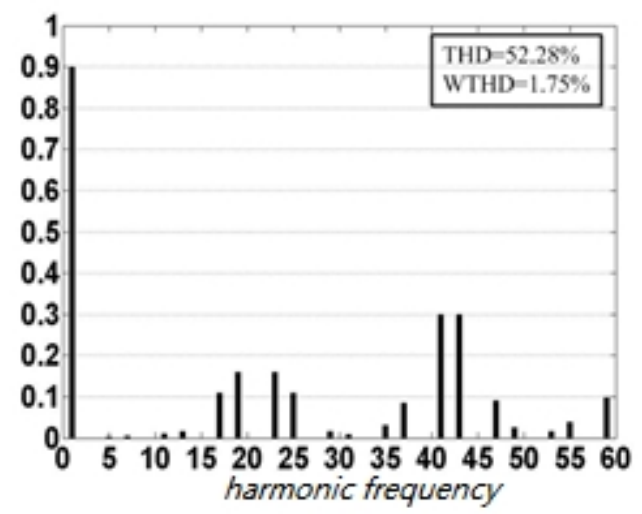

Fig. 6. SVPWM phase current spectrum

\section{Performance analysis and comparison}

SPWM in the switching function is not zero at the moment, only the load of the two phase is connected to the circuit, but also because of the same but the direction of the carrier wave in the same direction, so the current carrier harmonics are completely offset. Because the reference wave phase difference is 120 degrees, the phase current harmonics are generated, which make the harmonic wave in the presence of a 3 frequency band. The SPWM method of the symmetric rule sampling is generated at the same time and the harmonic of the non 3 frequency band and the non 3 frequency band are also produced. Asymmetric regular sampling SPWM, although the odd times of the odd side band around the odd times of the carrier wave and even the second harmonic of the carrier wave, but the band harmonics generated by odd times and 3 times. Comparison of the performance of the two indicators, found that the difference is not significant.

SVPWM is essentially a rule sampling procedure. The real difference between regular sampling PWM and SVPWM lies in the zero vector position within each half cycle. For regular sampling, it is not possible to change because the zero vector position has been set at the start of the modulation. However for SVPWM, the situation is quite different. The position of zero vector can be made by human. Because of this difference, the SVPWM has a better output characteristic. Not only that, it can be found that the modulation ratio of M is SVPWM, the maximum value is about 1.15 times that of SPWM. Compared with the SPWM method, the current utilization rate of SVPWM is higher. In the end, we can find the SVPWM has the best harmonic characteristics though the performance index of SVPWM is compared with the performance index of the first three SPWM.

\section{Conclusion}

In this paper, we study the modulation technology of the key technologies of high performance current transducer. The characteristics of different modulation algorithms and the reasons for the formation of harmonic are analyzed in detail. SVPWM is compared to the SPWM method, the SVPWM has a better output characteristics and higher current utilization when the zero vector of the position can be artificially made. 


\section{References}

[1] Luongo C A. Superconducting Storage Systems: An Overview. IEEE Transactions on Magnetics, 2007, 32(4):2214

[2] Karasik V, Dixon K, Weber C, et al. SMES For Power Utility Applications: A Review of Technical and Cost Considerations. IEEE Transactions on Applied Superconductivity, 1999, 9(2):541

[3] Wiechmann E P, Aqueveque P, Burgos R, et al. On the Efficiency of Voltage Source and Current Source Inverters for High-Power Drives. IEEE Transactions on Industrial Electronics, 2008, 55(4): 1771

[4] Bao J Y, Li Y L, Bai Z H, et al. Research on Topology and PWM Control Method of a Three-phase Five-level Current Inverter. Proceedings of the CSEE, 2006, 26(9):71

[5] Lopes A C, Naguib M F. Space Vector Modulation for Low Switching Frequency Current Source Cnverters With Reduced Low-Order Noncharacteristic Harmonics. IEEE Transactions on Power Electronics, 2009, 24(4):903

[6] Shang J, Yun W L. A Space-Vector Modulation Method for Common-Mode Voltage Reduction in Current-Source Converters. IEEE Transactions on Power Electronics, 2013, 29(1):374

[7] Dai J Y, Lang Y Q, Wu B, et al. Multisampling SVM Scheme for Current Source Converters With Superior Harmonic Performance. IEEE Transactions on Power Electronics, 2009, 24(11):2436

[8] Li J L, Lin P, Wang C Y, et al. Research on Current-Source Converter with Carrier Phase Shifted SPWM for Active Power Filter. Proceedings of the CSEE, 2003, 23(10):99

[9] Bai Z H, Ruan X B, Zhang Z C. A Generic Six-Step Direct PWM (SS-DPWM) Scheme for Current Source Converter. IEEE Transactions on Power Electronics, 2010, 25(3):659

[10]Bai Z H, Zhang Z C, Ruan X B. A Natural Soft-Commutation PWM Scheme for Current Source Converter and Its Logic Implementation. IEEE Transactions on Industrial Electronics, 2011, 58(7):2772

[11]Bai J, Lu S Q, Liu J. A novel PWM technology based on high-power current source inverter. Advanced Materials Research, 2014, 960:910

[12] Wang X, Ooi B T. Unity PF Current-Source Rectifier Based on Dynamic Trilogic PWM. IEEE Transactions on Power Electronics, 1993, 8(3):288

[13] Wu Wenxuan, Dan Yue Wu, Lin Yan, et. Current type inverter inter harmonic analysis and application of model construction [J]. Transactions of China Electrotechnical Society, 2010,25 (2): 1-5. (2) In Chinese

[14]Bai jing, Jian Liu, Lu Shiqi. A hybrid sequence modulation technique for common-mode voltage reduction in current source converters[J]. Advanced Materials Research, 2014(945-949) 